\title{
SURFACE DAMAGE TO COBALT-CHROME FEMORAL
}

\section{HEAD PROSTHESES}

\author{
MURALI JASTY, CHARLES R. BRAGDON, KYLA LEE, AMY HANSON, WILLIAM H. HARRIS
}

From Massachusetts General Hospital and Harvard Medical School, Boston, USA

Fifty-four cobalt-chrome alloy femoral heads were retrieved at revision surgery or at post-mortem; 23 came from uncemented, eight from hybrid and 23 from cemented hip arthroplasties. The uncemented and hybrid implants had porous coating, metal backing and modular femoral heads; with one exception none of the cemented implants had any of these.

Twenty-five of the 31 heads from uncemented and hybrid arthroplasties, and 11 of the 23 heads from cemented arthroplasties showed surface damage involving more than $25 \%$ of the surface. Scanning electron microscopy revealed multidirectional fine scratches $1 \mu \mathrm{m}$ to $10 \mu \mathrm{m}$ in depth and width which appeared to have been made by fine, hard particles.

There was a higher rate of such damage in the uncemented and hybrid arthroplasties than in the cemented implants, suggesting that the abrasive particles were mainly released from the metal, rather than from the cement or polyethylene components of the implants.

J Bone Joint Surg [Br] 1994; 76-B:73-7.

Received 13 January 1993; Accepted 22 April 1993

One of the most serious problems in total arthroplasty is wear of the polyethylene surfaces. Wear particles liberated into the surrounding soft tissues can produce a foreignbody granulomatous reaction and cause periprosthetic osteolysis and aseptic loosening (Harris et al 1976; Goldring et al 1986; Jasty et al 1986; Anthony et al 1990; Maloney et al 1990; Willert, Bertram and Buchhorn 1990; Schmalzried et al 1992).

Several factors influence the wear of polyethylene in total joint replacements: the chemical and physical properties of the polyethylene, the magnitude of the contact

M. Jasty, MD, Clinical Assistant, Professor of Orthopaedic Surgery

C. R. Bragdon, BS, Senior Research Technologist

K. Lee, BS, Research Assistant

A. Hanson, BA, Research Assistant

W. H. Harris, MD, Chief, Hip and Implant Unit

Orthopaedic Biomechanics Laboratory, Massachusetts General Hospital, Boston, Massachusetts 02114, USA.

Correspondence should be sent to Dr M. Jasty.

(C)1994 British Editorial Society of Bone and Joint Surgery $0301-620 X / 94 / 1654 \$ 2.00$ stresses, and the number of loading cycles (Rostoker 1978; Rose and Radin 1982; Weightman et al 1991). The surface hardness, surface finish and chemical composition of the metal femoral head also have their effect. Recently, several authors have reported early wear of titanium-alloy femoral heads (Agins et al 1988; Lombardi et al 1989; McKellop and Rostlund 1990); surface damage of stainless-steel heads has been described and attributed to entrapment of cement or bone particles in the artificial joint (Isaac et al 1986, 1987; Caravia et al 1990). The susceptibility of cobalt-chrome alloy heads to surface wear has not been addressed.

We examined 54 cobalt-chrome femoral heads retrieved at revision surgery or at post-mortem by detailed microscopic and metallurgical studies.

\section{MATERIALS AND METHODS}

Forty-nine cobalt-chrome femoral heads were retrieved at revision arthroplasty and five at post-mortem (Table I). Twenty-three of the arthroplasties had been performed with cement and 23 without. The other eight were hybrid arthroplasties with an uncemented acetabular component and a cemented femoral component. In no case was there catastrophic wear of the polyethylene component or dislocation of the femoral head. Details of the retrieved implants are given in Table I.

Most of the uncemented components were porous coated either with cobalt-chrome beads or titanium fibre mesh. All the cemented femoral components were made of cobalt-chrome alloys. All the hybrid total hip arthroplasties had Harris-Galante uncemented acetabular components and cemented Precoat femoral components of cobalt-chrome alloy (Zimmer, Warsaw, Indiana).

The heads used with cemented femoral components were manufactured before 1982; those used with uncemented and hybrid femoral components were manufactured after 1982. Their diameters varied from $22 \mathrm{~mm}$ to $32 \mathrm{~mm}$.

In the uncemented and hybrid groups all the heads were modular, attached to the femoral stems by tapered necks; each of the femoral components in the cemented group was manufactured in one piece. The uncemented femoral and acetabular components were all porous coated; the cemented femoral components all had a gritblasted finish. The uncemented acetabular components 
Table I. Details of 54 patients whose femoral heads were examined for abrasive wear

\begin{tabular}{|c|c|c|c|c|c|c|c|c|c|c|c|}
\hline \multirow[b]{2}{*}{ Case } & \multirow{2}{*}{$\begin{array}{l}\text { Age } \\
(\mathbf{y r})\end{array}$} & \multirow[b]{2}{*}{ Sex } & \multirow[b]{2}{*}{ Side } & \multirow{2}{*}{$\begin{array}{l}\text { Weight } \\
\text { (kg) }\end{array}$} & \multirow{2}{*}{$\begin{array}{l}\text { Duration } \\
(\mathbf{y r})\end{array}$} & \multirow[b]{2}{*}{ Disease* } & \multirow[b]{2}{*}{ Reason for removal } & \multicolumn{2}{|c|}{ Type of implant ${ }^{\dagger}$} & \multirow{2}{*}{$\begin{array}{l}\text { Head wear } \\
\text { (per cent) }\end{array}$} & \multirow{2}{*}{$\begin{array}{l}\text { Method of } \\
\text { retrieval } \neq\end{array}$} \\
\hline & & & & & & & & Acetabular & Femoral & & \\
\hline \multicolumn{12}{|c|}{ Uncemented } \\
\hline 1 & 61 & M & $\mathbf{R}$ & 97 & 3.0 & OA & Lysis & HG & HG & 50 & $\mathbf{S}$ \\
\hline 2 & 44 & $\mathbf{F}$ & $\mathrm{L}$ & 68 & 3.9 & AVN & Loosening & HG & HG & 30 & $S$ \\
\hline 3 & 50 & $\mathbf{M}$ & L & 54 & 5.0 & $\mathrm{OA}$ & Sepsis/loosening & HG & PCA & 100 & $\mathbf{S}$ \\
\hline 4 & 61 & $\mathbf{M}$ & L & 95 & 4.0 & OA & Dislocation & HG & HG & 30 & $\mathbf{S}$ \\
\hline 5 & 59 & $\mathbf{M}$ & $\mathrm{L}$ & 82 & 5.8 & OA & Lysis & HG & HG & 50 & $\mathbf{S}$ \\
\hline 6 & 41 & $\mathbf{M}$ & $\mathbf{R}$ & 70 & 6.0 & AVN & Lysis & HG & HG & 50 & $\mathrm{~S}$ \\
\hline 7 & 61 & $\mathbf{M}$ & $\mathbf{R}$ & 95 & 4.0 & $\mathrm{OA}$ & Loosening & HG & HG & 40 & $\mathbf{S}$ \\
\hline 8 & 38 & $\mathbf{M}$ & $\mathbf{R}$ & 88 & 1.6 & TOA & Loosening & HG & HG & 50 & $\mathbf{S}$ \\
\hline 9 & 64 & $\mathrm{~F}$ & $\mathbf{R}$ & 82 & 6.0 & $\mathrm{OA}$ & Lysis & PCA & PCA & 30 & $\mathbf{S}$ \\
\hline 10 & 60 & $\mathbf{M}$ & L & 75 & 7.0 & OA & Loosening/ysis & HG & HG & 30 & $\mathbf{S}$ \\
\hline 11 & 4.3 & $\mathbf{M}$ & $\mathrm{L}$ & 104 & 4.8 & OA & Loosening & PCA & PCA & 25 & $\mathbf{S}$ \\
\hline 12 & 33 & $\mathrm{~F}$ & $\mathrm{~L}$ & 49 & 3.0 & $\mathrm{CDH}$ & Loosening & Omniflex & Omniflex & 25 & $\mathrm{~S}$ \\
\hline 13 & 64 & $\mathbf{F}$ & L. & 72 & 5.3 & AVN & Lysis & HG & HG & 50 & $\mathrm{~S}$ \\
\hline 14 & 49 & $\mathbf{M}$ & $\mathbf{R}$ & 72 & 0.8 & OA & Sepsis & Omniflex & Omniflex & 50 & $\mathrm{~S}$ \\
\hline 15 & 70 & $\mathbf{M}$ & $\mathrm{L}$ & 63 & 5.4 & AVN & Lysis & HG & PCA & 75 & $\mathrm{~S}$ \\
\hline 16 & 61 & $\mathrm{~F}$ & $\bar{R}$ & 49 & 6.0 & $\mathrm{CDH}$ & Lysis/loosening & HG & HG & 70 & $\mathrm{~s}$ \\
\hline 17 & 6.3 & $\mathrm{~F}$ & I. & 84 & 5.0 & TOA & Loosening/ysis & HG & HG & 50 & $\mathbf{S}$ \\
\hline 18 & 42 & $\mathbf{M}$ & $\mathbf{R}$ & 59 & 0.8 & AVN & Loosening & OPTI-FIX & OPTI-FIX & 60 & $\mathbf{S}$ \\
\hline 19 & 41 & $\mathbf{M}$ & L & 70 & 6.0 & AVN & Lysis & HG & HG & 0 & $\mathbf{S}$ \\
\hline 20) & 59 & $F$ & L & 95 & 4.0 & OA & Loosening & HG & HG & 50 & $\mathrm{~S}$ \\
\hline 21 & 72 & $\mathrm{~F}$ & $\mathbf{R}$ & 66 & 4.3 & $\mathrm{CDH}$ & Loosening & HG & AML & 95 & $\mathbf{S}$ \\
\hline 22 & 72 & $\mathrm{~F}$ & L & 59 & 1.0 & $\mathrm{OA}$ & Post-mortem & AML & AML & 5 & PR \\
\hline 23 & 78 & $\mathbf{M}$ & $\mathbf{R}$ & 78 & 7.2 & OA & Post-mortem & HG & HG & 90 & PR \\
\hline \multicolumn{12}{|c|}{ Cemented } \\
\hline 1 & 84 & $\mathbf{F}$ & $\mathbf{R}$ & 55 & 18.0 & OA & Loosening & MUL & MUL & 5 & $\mathbf{S}$ \\
\hline 2 & 75 & $\mathrm{~F}$ & $\mathrm{~L}$ & 61 & 16.6 & AVN & Loosening & Harris & Harris & 5 & $\mathrm{~S}$ \\
\hline 3 & 64 & $\mathbf{F}$ & $\mathbf{R}$ & 59 & 18.8 & $\mathrm{CDH}$ & Lysis & Harris & Harris & 0 & $\mathbf{S}$ \\
\hline 4 & 72 & $\mathbf{F}$ & $\mathbf{R}$ & 70 & 11.5 & $\mathrm{CDH}$ & Loosening & MM & MM & 0 & $\mathbf{S}$ \\
\hline 5 & 39 & $\mathbf{F}$ & $\mathrm{L}$ & 63 & 10.8 & $\mathrm{OA}$ & Loosening & Harris & Harris & 30 & $\mathbf{S}$ \\
\hline 6 & 42 & $\mathbf{F}$ & L & 57 & 5.7 & $\mathrm{CDH}$ & Loosening & MUL & MUL & 70 & $\mathbf{S}$ \\
\hline 7 & 60 & $\mathbf{M}$ & $\mathrm{L}$ & 82 & 4.0 & $\mathrm{OA}$ & Loosening & MUL & MUL & 50 & $\mathbf{S}$ \\
\hline 8 & 57 & $\mathbf{M}$ & $\mathbf{R}$ & 91 & 7.0 & OA & Broken stem & Harris & Harris & 100 & $\mathbf{S}$ \\
\hline 9 & 68 & $\mathbf{M}$ & $\mathbf{R}$ & 86 & 6.0 & OA & Loosening/ysis & MUL & MUL & 0 & $\mathbf{S}$ \\
\hline 10 & 74 & $\mathbf{M}$ & L & 80 & 6.5 & OA & Loosening & MUL & MUL & 0 & $\mathrm{~S}$ \\
\hline 11 & 75 & $\mathbf{M}$ & $\mathbf{R}$ & 95 & 11.2 & OA & Loosening/lysis & AT & AT & 25 & $\mathbf{S}$ \\
\hline 12 & 51 & $\mathbf{M}$ & $\mathbf{R}$ & 83 & 16.0 & TOA & Heterotopic ossification & MB & Harris & 60 & $\mathrm{~S}$ \\
\hline 13 & 63 & $\mathrm{~F}$ & $\mathbf{R}$ & 58 & 13.0 & $\mathrm{CDH}$ & Loosening & Harris & Harris & 10 & $\mathrm{~S}$ \\
\hline 14 & 29 & $\mathbf{F}$ & L & 30 & 10.0 & RA & Loosening/lysis & AT & AT & 5 & $\mathbf{S}$ \\
\hline 15 & 73 & $\mathbf{F}$ & $\mathbf{R}$ & 75 & 1.0 & $O A$ & Loosening & HD2 & HD2 & 20 & PR \\
\hline 16 & 55 & $\mathrm{~F}$ & $\mathrm{~L}$ & 54 & 12.1 & $\mathrm{CDH}$ & Loosening & CAD & CAD & 60 & $\mathrm{~s}$ \\
\hline 17 & 71 & $\mathrm{~F}$ & $\vec{L}$ & 67 & 4.6 & OA & Loosening & Harris & Harris & 60 & $\mathrm{~S}$ \\
\hline 18 & 47 & $\mathrm{~F}$ & $\mathbf{R}$ & 59 & 6.0 & $\mathrm{CDH}$ & Loosening & MM & MM & 50 & PR \\
\hline 19 & 65 & M & $\mathbf{R}$ & 79 & 4.3 & $\mathrm{OA}$ & Loosening & AT & AT & 0 & $\mathrm{~S}$ \\
\hline 20 & 69 & $\mathbf{M}$ & L & 86 & 3.0 & TOA & Loosening & CAD & MUL & 50 & $\mathbf{S}$ \\
\hline 21 & 69 & $\mathrm{~F}$ & $\vec{R}$ & 68 & 10.0 & $\mathrm{OA}$ & Loosening & MUL & MUL & 60 & $\mathbf{S}$ \\
\hline 22 & 46 & $\mathbf{M}$ & $\mathbf{R}$ & 82 & 2.0 & AVN & Loosening & MUL & MUL & 5 & S \\
\hline 23 & 71 & $\mathbf{M}$ & $\mathbf{R}$ & 77 & 13.5 & OA & Loosening & $\mathrm{CAD}$ & $\mathrm{CAD}$ & 50 & $\mathbf{S}$ \\
\hline \multicolumn{12}{|c|}{ Hybrids } \\
\hline 1 & 73 & $\mathbf{M}$ & L. & 82 & 1.6 & OA & Sepsis & HG & PRE & 40 & $\mathbf{S}$ \\
\hline 2 & 66 & $\mathrm{~F}$ & $\mathbf{R}$ & 59 & 5.0 & $\mathrm{CDH}$ & Sepsis & HG & PRE & 50 & $\mathbf{S}$ \\
\hline 3 & 56 & $\mathbf{M}$ & $\mathbf{R}$ & 95 & 1.0 & $\mathrm{OA}$ & Sepsis & HG & PRE & 50 & $\mathbf{S}$ \\
\hline 4 & 58 & $\mathbf{M}$ & $\mathbf{R}$ & 77 & 0.8 & OA & Loosening & HG & PRE & 20 & $\mathbf{S}$ \\
\hline 5 & 71 & $\mathrm{~F}$ & $\mathbf{R}$ & 52 & 2.9 & $O A$ & Loosening & HG & PRE & 90 & $\mathbf{S}$ \\
\hline 6 & 72 & $\mathbf{M}$ & $\mathbf{R}$ & 76 & 1.0 & OA & Post-mortem & HG & PRE & 20 & PR \\
\hline 7 & 56 & $\mathbf{F}$ & $\mathbf{R}$ & 54 & 4.9 & RA & Loosening & HG & PRE & 40 & S \\
\hline 8 & 65 & M & $\mathbf{R}$ & 82 & 4.0 & TOA & Loosening & HG & PRE & 80 & $\mathbf{S}$ \\
\hline
\end{tabular}

* $\mathrm{OA}=$ osteoarthritis; $\mathrm{AVN}=$ avascular necrosis; $\mathrm{TOA}=$ post-traumatic osteoarthritis; $\mathrm{CDH}=$ congenital dislocation of the hip; $\mathrm{RA}=$ rheumatoid arthritis † HG=Zimmer, Warsaw, Indiana; PCA=Howmedica, Rutherford, New Jersey; Omniflex=Osteonics, Allendale, New Jersey; OPTl-FIX=Smith \& Nephew Richards, Memphis, Tennessee; AML=DePuy, Warsaw, Indiana; MUL=Muller curved stem, Howmedica; Harris=Howmedica; MM=Micromini, Howmedica; AT=Aufranc Turner, Howmedica; HD2=Howmedica; MB=Harris metal bracket, Howmedica; CAD=Computer Assisted Design, Howmedica; PRE=Harris Precoat, Zimmer

$\ddagger S=$ retrieved at surgery; $P R=$ retrieved at post-mortem

were all metal-backed; only one of the cemented acetabular components was metal-backed. Of the 31 uncemented acetabular components, 25 were fixed with screws.

All the heads were carefully removed to avoid damage during extraction and were examined by the naked eye and through a dissecting microscope at $10 \times$ magnification. Loss of the mirror finish and the presence of scratch marks were recorded. When surface scratching was present, the percentage of the femoral head involved was estimated.

The femoral heads were then washed with non- 


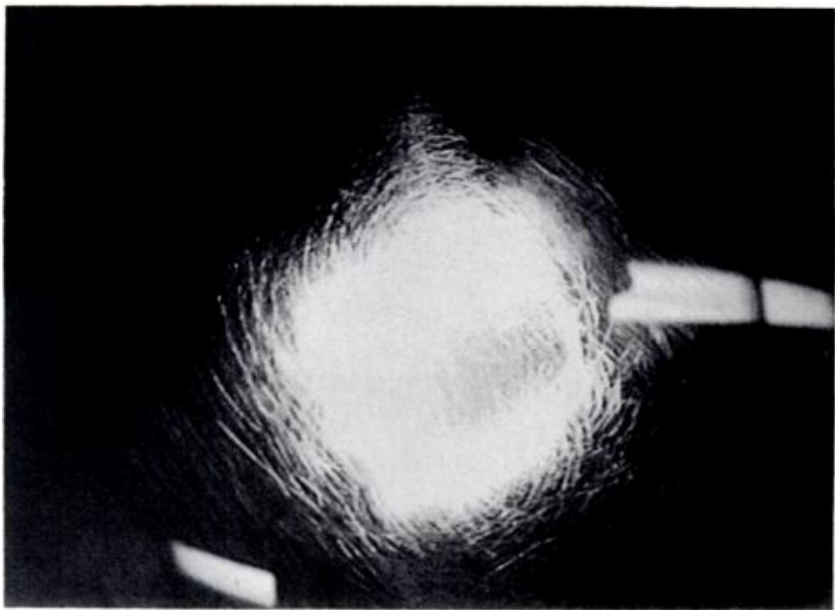

Fig. 1

A cobalt-chrome femoral head photographed by reflected light to show surface damage in the form of multiple scratches.

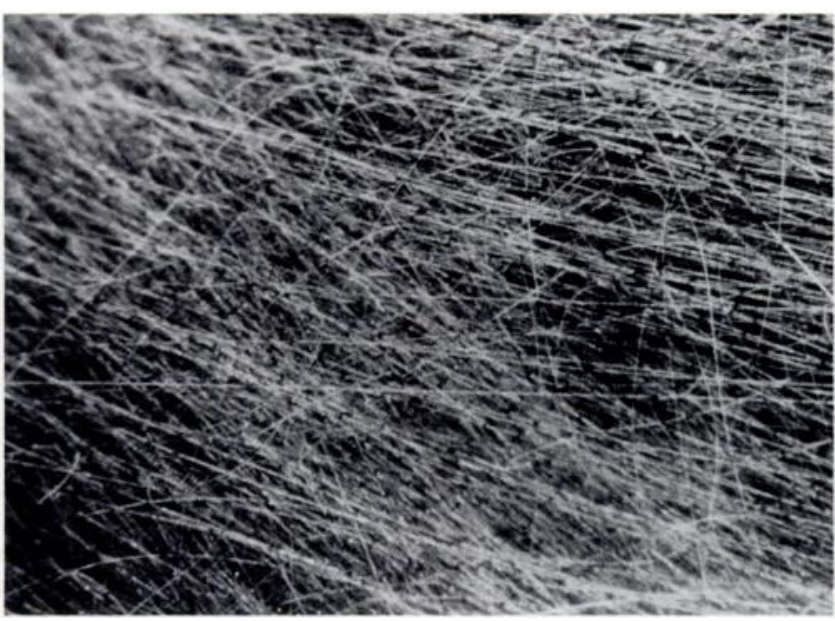

Fig. 2a younger (mean age 56 years) than those in the cemented group (mean age 62 years) and the hybrid group (mean age 65 years). The duration of service of the prosthesis was greater in the cemented group (mean 9.2 years, range 1 to 18.8 ) than in the uncemented group (mean 4.3 years, range 0.75 to 7.2 ).

Of the 54 heads, 48 showed some evidence of surface damage. To the naked eye this appeared as slight loss of the mirror finish. The affected areas were usually discrete, measuring 1 to $20 \mathrm{~mm}^{2}$ usually on the upper surface (Fig. 1).

Under the microscope, these areas had numerous fine scratches running in all directions (Fig. 2a). Only an occasional fine scratch was seen in areas which retained their mirror finish (Fig. 2b). There was usually a sharp demarcation between areas with many scratches and those without.

Most of the scratches were 0.1 to $5.0 \mu \mathrm{m}$ wide and

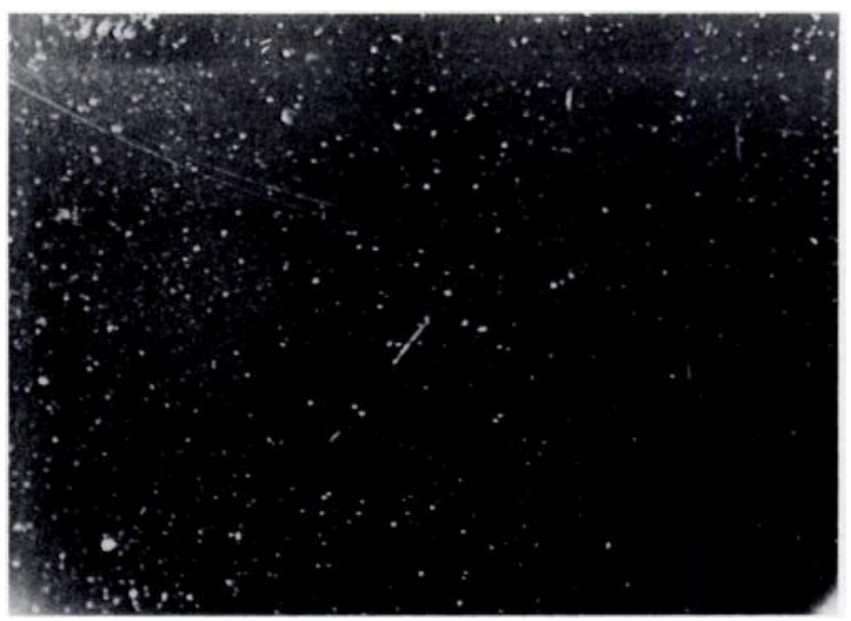

Fig. 2b

Through the dissecting microscope multiple fine scratches (a) are seen running in all directions (magnification $\times 16$ ). An area without significant damage showed multiple voids (b) but minimal scratching.

abrasive soap and water, cleaned with $100 \%$ alcohol, dried and re-examined under a scanning electron microscope (Cambridge Instruments, Cambridge, UK) at low, medium and high power. The average depth and width of the scratches were recorded and their morphology was compared with that reported by Isaac et al (1987) in stainless-steel femoral heads. The unscratched areas were also examined.

Four unused femoral heads (two PCA and two Precoat) were obtained and their surfaces were examined by the same methods.

\section{RESULTS}

There were no significant differences in the sex distribution, body-weight, initial diagnosis, femoral-head size, or the reason for revision in the patients in the three groups (Table I). Those in the uncemented group were slightly
0.1 to $2.0 \mu \mathrm{m}$ deep, although some larger and some smaller scratches were seen (Figs 3 and 4). Their morphology was similar to that of the scratches produced by Isaac et al (1987) by wearing stainless-steel surfaces against polyethylene containing bone-cement particles. The scratches often had sharp edges with heaped-up boundaries (Fig. 4), suggesting that they were caused by three-body abrasive wear from hard particles trapped in the joint. Small pits about $2 \mu \mathrm{m}$ in diameter were occasionally seen due to residual porosities left in the metal during the manufacturing process. In spite of the extensive scratching, no loose particles were found in these indentations. The areas which did not show scratches appeared smooth even at a magnification of $2000 \times$.

Surface damage was more common, more extensive and occurred after shorter periods of service in uncemented and hybrid arthroplasties than in those fixed with cement. Of the 31 heads used in uncemented or hybrid 
arthroplasties, 25 had surface damage involving more than $25 \%$ of the head but only 11 of the 23 heads used in cemented arthroplasties showed such damage. This difference is statistically significant at $95 \%$ confidence intervals using the two-by-two chi-squared test. Five of the 23 heads in the cemented group had no appreciable damage, and four had $5 \%$ or less of the head scratched, despite long periods of service, up to 18 years in one case. By contrast, only one of the 23 heads in the uncemented group, and none of the eight heads in the hybrid group, had less than $20 \%$ of the surfaces damaged, although the duration of follow-up was shorter. Moderate (one- to twothirds of the surface) or severe damage (more than twothirds of the surface) was found in 18 of the 23 heads in

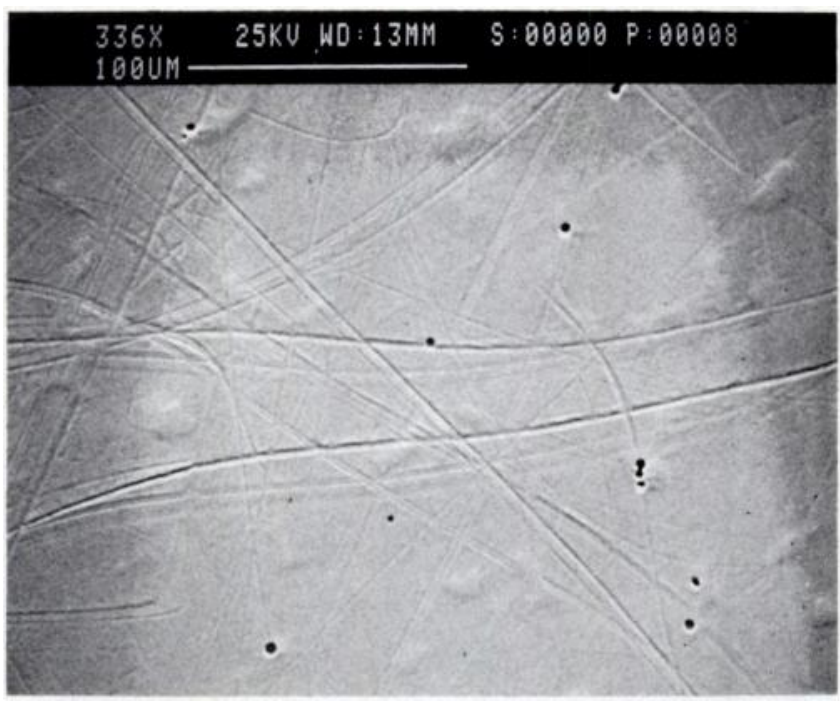

Fig. 3

Low-power scanning electron micrograph of several scratches at low contrast. The dark spots are voids in the femoral head produced during manufacturing. the uncemented group, in six of the eight heads in the hybrid group, and in 11 of the 23 in the cemented group. There were, however, no differences in the morphological appearance of the scratches in the three groups.

The unused femoral heads generally had a mirrorlike finish with no visible surface damage. Examination under the electron microscope at high power showed only small pits from residual porosity and no sharp-edged scratches were seen.

\section{DISCUSSION}

Cobalt-chrome femoral heads are thought to resist wear better than titanium or stainless-steel heads because of their hard surface. We have shown, however, that surface damage in the form of multiple fine scratches does occur on these heads and that the appearance of the scratches suggests that they are produced by entrapped particles in the joint. The surface damage occurs more frequently, earlier and more extensively in uncemented and hybrid than in cemented arthroplasties. This difference may be due to several factors such as modularity, porous coating and metal backing.

Isaac et al (1986) found that the heads of retrieved stainless-steel Charnley components showed fine multidirectional scratches up to $8 \mu \mathrm{m}$ in width. They suggested that entrapped bone-cement particles were responsible. In subsequent experiments (Isaac et al 1987), they demonstrated that clusters of barium or zirconium particles from the radiographic contrast material in the acrylic cement were the cause of the abrasions.

We found similar scratches, however, in arthroplasties in which cement had not been used. In these heads the abrasive particles must have come from the metal of the uncemented devices themselves, or less probably from silica particles in the polyethylene.

Extensive damage to the surface of femoral heads

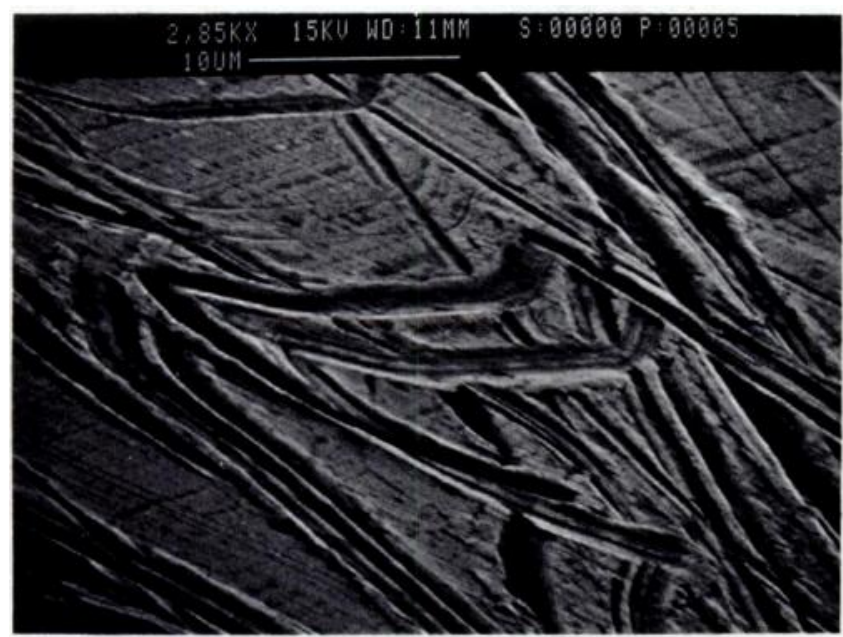

Fig. 4a

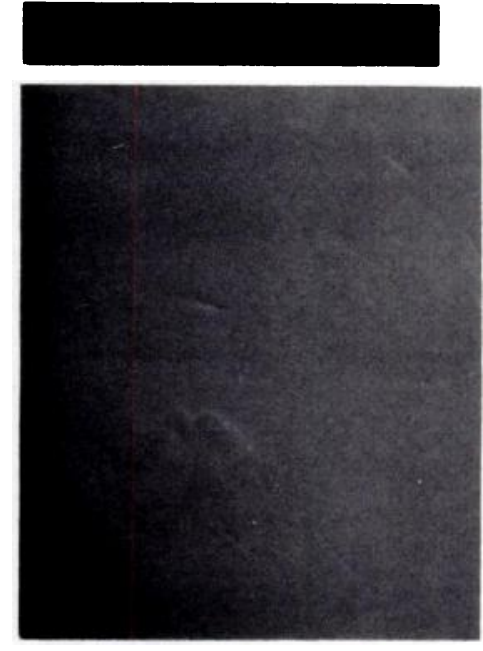

Fig. $4 \mathrm{~b}$

High-power scanning electron micrographs showing details of the scratches. a) There is deposition of material at the edges of the grooves. b) A new femoral head is shown for comparison. 
made of titanium-based alloys has been reported by Lombardi et al (1989). Titanium alloys are more prone to abrasive wear by entrapped particles because of the softness of the alloy and because the oxide film layer on its surface may undergo repeated disruption. The titaniumoxide particles so liberated may themselves further accelerate wear. Retrieved titanium heads have often shown numerous coarse multidirectional scratches over most of the surface and in some cases the wear was so extensive that the head was no longer spherical. None of the cobalt-chrome heads which we examined showed such severe wear. They all remained spherical, and the scratches were fine and usually confined to small areas.

Our observations suggest three-body wear from particles released from outside the articulation, but other possibilities must also be considered. Using radioactive tracers, Rabinowicz and Shooter (1952) have shown that there is some transfer of metal to polyethylene during the process of wear. These small metal particles could then act as an abrasive between the head and the socket. There are also impurities in the polyethylene such as silica which could cause scratches on the femoral head.

The observation that the frequency and severity of femoral head damage were greater in the uncemented and hybrid arthroplasties supports the theory that the most important abrasive agents are entrapped metal particles. The porous-surfaced components used in uncemented arthroplasty expose large areas of metal to the bone, and may generate larger quantities of fine metallic debris due to corrosion or fretting. Furthermore, such components are usually modular either at the neck-head junction or between the acetabular cup and its metal liner. Fretting or corrosion at these sites is known to occur and may be sources of fine metal debris (Mathiesen et al 1991).

The effects of femoral-head surface damage may have importance for wear of the polyethylene cup. The three-body wear mechanism which caused the fine scratches may be expected to damage the polyethylene as well (McKellop and Rostlund 1990). More importantly, a damaged femoral head may accelerate two-body abrasive wear of the cup. The effect of counterface roughness, however, on the wear rate of polyethylene is uncertain. Rabinowicz (1966) suggested that with time the sharp edges of the scratches may become smooth, that the troughs fill in with debris, and that this wearing-in process may eventually decrease the ill-effects of a scratched surface.

The wear rate of polyethylene depends on several variables, such as its molecular weight and crystallinity, the method of manufacture, the effects of radiation and oxidation, and the magnitude of the contact stresses (Kurth et al 1988; Rose and Radin 1982). One of the most important controllable variables, however, is abrasive wear and our study shows that this occurs in vivo even when cobalt-chrome heads are used.
This work was supported by the William H. Harris Foundation.

One or more of the authors have received or will receive benefits for personal or professional use from a commercial party related directly or indirectly to the subject of this article. In addition benefits have also been or will be directed to a research fund, foundation, educational institution, or other non-profit institution with which one or more of the authors is associated.

\section{REFERENCES}

Agins HJ, Alcock NW, Bansal M, et al. Metallic wear in failed titaniumalloy total hip replacements: a histological and quantitative analysis. $J$ Bone Joint Surg [Am] 1988; 70-A:347-56.

Anthony PP, Gie GA, Howie CR, Ling RSM. Localised endosteal bone lysis in relation to the femoral components of cemented total hip arthroplasties. J Bone Joint Surg [Br] 1990; 72-B:971-9.

Caravia L, Dowson D, Fisher J, Jobbins B. The influence of bone and bone cement debris on counterface roughness in sliding wear tests of ultra-high molecular weight polyethylene on stainless steel. Proc Inst Mech Eng 1990; 204:65-70.

Goldring SR, Jasty M, Roelke MS, et al. Formation of a synovial-like membrane at the bone-cement interface: its role in bone resorption and implant loosening after total hip replacement. Arthritis Rheum 1986 29:836-42.

Harris WH, Schiller AL, Scholler JM, Freiberg RA, Scott R. Extensive localized bone resorption in the femur following total hip replacement. J Bone Joint Surg [Am] 1976; 58-A:612-8.

Isaac GH, Atkinson JR, Dowson D, Wroblewski BM. The role of cement in long-term performance and premature failure of Charnley low friction arthroplasties. Eng Med 1986; 15(1):19-22.

Isaac GH, Atkinson JR, Dowson D, et al. The causes of femoral head roughening in explanted Charnley hip prostheses. Eng Med 1987; 16(3):167-73.

Jasty MJ, Floyd WE III, Schiller AL, Goldring SR, Harris WH. Localized osteolysis in stable, non-septic total hip replacement. J Bone Joint Surg [Am] 1986; 68-A:912-9.

Kurth M, Eyerer P, Ascherl R, Dittel K, Holz U. An evaluation of retrieved UHMWPE hip joint cups. J Biomater Appl 1988; 3 33-51.

Lombardi AV, Mallory TH, Vaughan BK, Drouillard P. Aseptic loosening in total hip arthroplasty secondary to osteolysis induced by wear debris from titanium-alloy modular femoral heads. J Bone Joint Surg [Am] 1989; 71-A:1337-42.

Maloney WJ, Jasty M, Harris WH, Galante JO, Callaghan JJ. Endosteal erosion in association with stable uncemented femoral components. $J$ Bone Joint Surg [Am] 1990; 72-A:1025-34.

Mathiesen EB, Lindgren JU, Blomgren GGA, Reinholt FP. Corrosion of modular hip prostheses. J Bone Joint Surg [Br] 1991; 73-B:569-75.

McKellop HA, Rostlund TV. The wear behavior of ion-implanted Ti-6Al4V against UHMW polyethylene. J Biomed Mater Res 1990; 24:1413-25.

Rabinowicz E. Friction and wear of materials. New York, etc: John Wiley and Sons, 1966.

Rabinowicz E, Shooter KV. The transfer of metal to plastics during sliding. Procs Physical Society 1952; B, LXV:671-3.

Rose RM, Radin EL. Wear of polyethylene in the total hip prosthesis. Clin Orthop 1982; 170:107-15.

Rostoker W. The appearances of wear on polyethylene: a comparison of in vivo and in vitro wear surfaces. $J$ Biomed Mater Res 1978; 12 : 317-35.

Schmalzried TP, Kwong LM, Jasty M, et al. The mechanism of loosening of cemented acetabular components in total hip arthroplasty: analysis of specimens retrieved at autopsy. Clin Orthop 1992; 274:60-78.

Weightman B, Swanson SAV, Isaac GH, Wroblewski BM. Polyethylene wear from retrieved acetabular cups. J Bone Joint Surg [Br] 1991; 73-B:806-10.

Willert HG, Bertram H, Buchorn GH. Osteolysis in alloarthroplasty of the hip: the role of ultra-high molecular weight polyethylene wear particles. Clin Orthop 1990; 258:95-107.

Wroblewski BM. Wear and loosening of the socket in the Charnley lowfriction arthroplasty. Orthop Clin North Am 1988; 19:627-30. 